\title{
Phytochemical Screening and Cytotoxic Properties of Ethanolic Extract of Young and Mature Khat Leaves
}

\author{
Rashad Alsanosy, ${ }^{1,2}$ Hassan A. Alhazmi iD, ${ }^{1,3}$ Shahnaz Sultana, ${ }^{4}$ Ashraf N. Abdalla, ${ }^{5,6}$ \\ Yassin Ibrahim, ${ }^{7}$ Mohammed Al Bratty $\left(0,{ }^{3}\right.$ David Banji, ${ }^{8}$ Ibrahim Khardali, \\ and Asaad Khalid $\oplus^{1,6}$ \\ ${ }^{1}$ Substance Abuse and Toxicology Research Center, Jazan University, Jazan, Saudi Arabia \\ ${ }^{2}$ Faculty of Medicine, Jazan University, P.O. Box 114, Jazan 45142, Saudi Arabia \\ ${ }^{3}$ Department of Pharmaceutical Chemistry, College of Pharmacy, Jazan University, P.O. Box 114, Postal Code 45142, Jazan, \\ Saudi Arabia \\ ${ }^{4}$ Department of Pharmacognosy, College of Pharmacy, Jazan University, P.O. Box 114, Jazan 45142, Saudi Arabia \\ ${ }^{5}$ Department of Pharmacology and Toxicology, Faculty of Pharmacy, Umm Al-Qura University, Makkah 21955, Saudi Arabia \\ ${ }^{6}$ Medicinal and Aromatic Plants Research Institute, National Center for Research, P.O. Box: 2424, Khartoum-11111, Sudan \\ ${ }^{7}$ Faculty of Medicine, University of Tabuk, Tabuk, Saudi Arabia \\ ${ }^{8}$ Pharmacy Practice Research Unit, Department of Clinical Pharmacy, College of Pharmacy, Jazan University, P.O. Box 114, \\ Jazan 45142, Saudi Arabia \\ ${ }^{9}$ Poison Control and Medical Forensic Chemistry Center, Ministry of Health, Jazan, Saudi Arabia
}

Correspondence should be addressed to Asaad Khalid; drasaad@gmail.com

Received 2 September 2019; Revised 14 December 2019; Accepted 6 January 2020; Published 9 March 2020

Academic Editor: Maria C. Yebra-Biurrun

Copyright (c) 2020 Rashad Alsanosy et al. This is an open access article distributed under the Creative Commons Attribution License, which permits unrestricted use, distribution, and reproduction in any medium, provided the original work is properly cited.

The khat plant has been culturally used in many parts of Africa and the Arabian Peninsula for many years to induce psychostimulating effect. Because of the global wide-spreading nature, khat chewing is being considered as a universally growing problem. Catha abbottii, Catha edulis, and Catha transvaalensis are the three species of khat commonly chewed in Saudi Arabia and nearby regions. Khat users usually prefer to chew young leaves over mature ones due to the diverse effects produced by both. Though many of the constituents of khat leaves have been identified, the complete phytochemical profile of young and mature leaves was not performed or compared; also, no evidence is available to affirm the cytotoxicity of young or mature leaves. Therefore, this study aimed to investigate the phytochemical basis of the differential response of the young and mature leaves and to assess the cytotoxicity of young and mature khat leaves. Ethanolic extracts of young and mature leaves of three khat cultivars were subjected to GC-MS. Hierarchical cluster analysis revealed the existence of two major clusters. The extracts of young leaves were found to contain the maximum content of cathinone; however, methoxyamphetamine was found in only one extract of young leaves. Cytotoxicity investigations were also conducted on both types of leaves using three cancer cell lines, human breast adenocarcinoma, human ovary adenocarcinoma, and human colon adenocarcinoma and also normal human fetal lung fibroblast cell line was used. All extracts showed comparable cytotoxicity, $\mathrm{IC}_{50}$ ranging from $22-59 \mu \mathrm{g} / \mathrm{mL}$ on the cancer cells; however, we observed more cytotoxicity against normal cells $\left(\mathrm{IC}_{50}: 6-41 \mu \mathrm{g} / \mathrm{mL}\right.$ ). The predominant cytotoxicity on normal cells may pose many health hazards to khat consumers.

\section{Introduction}

Khat (Catha edulis) has culturally been used in many parts of Africa and the Arabian Peninsula, including Saudi Arabia and Yemen, but it is believed to be a globally growing problem [1-3]. Khat is mainly used to increase mental capacity [4], physical strength [5], and social entertainment [6] and enhance cheerfulness [7] and sexual orgasms [8]. The World Health Organization considers khat a drug of abuse since it causes a range of health problems [9]. There is a 
biological plausibility that chronic khat use may induce memory deficits and impair cognitive flexibility [10]. The differential patterns of memory deficits may reflect the differences in dose effect as well as time-dependent impairment [11]. Cathinone, a major constituent of khat, is structurally and functionally similar to amphetamine and cocaine. Cathinone caused the proliferation of gonadotrophs but decreased the lactotrophs and corticotrophs in anterior pituitary sections of animals in high dose and long-term exposure, while an effect of low dose on these cells was insignificant [12]. Few articles contribute to death among khat chewers to khat-induced heart failure, but several other studies have demonstrated that khat chewing has unfavorable cardiovascular effects [13]. Like amphetamine, the reflection of undesired actions of cathinone on the myocardium is observed through variations in heart rate, blood pressure, and vascular actions [14]. However, further studies are needed to address the risk factors in khat chewers that may explain khat-induced cardiotoxicity, cardiomyopathy, and heart failure.

Khat is reported to induce toxic hepatitis together with high-titer anti-nuclear antibody mimicking serologic patterns of autoimmune hepatitis and potentially associated with malignant and malignant oral disorders [15]. There are several pieces of evidence connecting khat chewing to genetic damage of the oral mucosa and cancer $[16,17]$ Esophageal and gastric carcinoma have been observed in khat chewers in both men and women in Yemen [18]. A similar review of oral cancers presenting over two years in the Asir region of Saudi Arabia showed strong circumstantial evidence linking the long-term use of khat with an increased rate of oral malignancies [19].

Khat young and mature leaves have a different degree of psycho-stimulating and variable biological properties. Khat chewers, however, prefer young leaves over mature for the more desired outcomes and fewer side effects. The young leaves are believed to possess higher central nervous system(CNS-) stimulating activities than the mature leaves. This difference in CNS-related activities could be due to the presence of the same constituents in both young and mature leaves; however, in different quantities or due to the presence of different constituents should be investigated. No assessment and comparison of the complete phytochemical profiles of young and mature leaves of Catha edulis has been conducted. Therefore, we have undertaken this study to investigate the phytochemical constituents of both young and mature khat leaves and to find out the degree of cytotoxicity of their selected extracts.

\section{Materials and Methods}

2.1. Collection of Plant Material. Young leaves (locally known as Nwaif) and mature leaves (locally known as Gafra) of three cultivars of khat (Catha edulis) locally known as Gaifi, Kofat, and Gahasha were collected from the Jazan region of Saudi Arabia and divided into two groups each: young Gaifi (N1), young Kofat (N2), and young Gahasha (N3) for young leaves and mature Gaifi (G1), mature Kofat (G2), and mature Gahasha (G3) for mature leaves. All khat varieties were identified by Dr. Yahiya Masrahi, Department of Botany, Faculty of Science, Jazan University. The collected leaves were washed, dried in the shade at room temperature, and powdered.

2.2. Preparation of Leaves Extract. All dried leaves powder (200 g each) was exhaustively extracted with $80 \%$ ethanol in the Soxhlet apparatus for 6 to 8 hours. Colored extracts were evaporated under reduced pressure to get brown viscous masses. Sample N1, N2, N3, G1, G2, and G3 were labeled and stored at $4^{\circ} \mathrm{C}$ in the dark for GC-MS analysis and cytotoxic activity.

2.3. GC-MS Analysis of the Extracts. GC-MS analyses of each extract were carried out on a Shimadzu Gas Chromatograph instrument (Shimadzu GCMS QP2010 with CTC GC PAL Liquid Injector Computer Software Loaded) fitted with a capillary column TR-5MS $(30 \mathrm{~m} \times 0.25 \mathrm{~mm})$, with film thickness $0.25 \mu \mathrm{m}$. The carrier gas was $\mathrm{He}$, with the flow rate of $1.2 \mathrm{~mL} / \mathrm{min}$. The initial temperature was kept at $70^{\circ} \mathrm{C}$ and then heated at a rate of $15^{\circ} \mathrm{C}$ per minute to $290^{\circ} \mathrm{C}$ and held for 16 minutes. The chromatography was coupled with a Shimadzu QP2010 Ultra MS detector, $70 \mathrm{eV}$.

2.4. Identification of Constituents. GC-MS identified the most constituents by comparing their retention indices with those of authentic standards available in the laboratory or with the retention indices, which were in close agreement with the reference. GC-MS achieved further identification. The fragmentation patterns of mass spectra were compared with those stored in the spectrometer database using the NIST08 and Wiley 9 built libraries.

2.5. Hierarchical Cluster Analysis (HCA). Due to significant variations of the contents of various types of khat leaves, the most abundant components from all samples were subjected to multivariate chemometric analysis. Hierarchical cluster analysis (HCA) was performed, and agglomeration and dendrograms were developed to assess the relationship between the components of the different types of extracted khat and to find out the proper classification of these studied samples and to detect the proper classification of khat types using SPPS software version 22.0.

2.6. Cell Culture. Three cancer cell lines, MCF7 (Michigan Cancer Foundation-7; human breast adenocarcinoma), A2780 (human ovary adenocarcinoma), and HT29 (human colon adenocarcinoma), were used in this study to assess the cytotoxicity of different ethanolic extracts. Besides, we also used MRC5, normal human fetal lung fibroblast. All cells were obtained from the ATCC (American Type Culture Collection). The three cancer cells were subcultured in the RPMI (Roswell Park Memorial Institute)-1640 media (10\% FBS, fetal bovine serum), while MRC5 was maintained in Eagles Minimum Essential Medium (EMEM, 10\% FBS), all at $37^{\circ} \mathrm{C}, 5 \% \mathrm{CO}_{2}$ and $100 \%$ relative humidity). 
2.7. Cytotoxicity Assay. As previously reported by Bkhaitan et al., the cytotoxic activity of the six extracts was evaluated by the MTT assay (MTT is the abbreviation for 3-(4,5-dimethylthiazol-2-yl)-2,5-diphenyltetrazolium bromide). The three cell lines and one normal fibroblast were separately cultured in a 96-well $\left(3 \times 10^{3} /\right.$ well $)$ and incubated at $37^{\circ} \mathrm{C}$ overnight. Final extract concentrations were $0,6.25,12.5,25$, 50 , and $100 \mu \mathrm{g} / \mathrm{mL}$ of DMSO (dimethyl sulfoxide) $0.1 \%$; $n=3$ ). Plates were incubated for $72 \mathrm{~h}$, followed by addition of MTT to each well. Then, the plates were incubated for $3 \mathrm{hr}$, the supernatant was aspirated, and DMSO was added to each well. Absorbance was read on the multiplate reader. The optical density of the purple formazan $\mathrm{A}_{550}$ is proportional to the number of viable cells. Extract concentration causing $50 \%$ inhibition $\left(\mathrm{IC}_{50}\right)$ compared to the control cell growth $(100 \%)$ was determined. GraphPad Software (GraphPad Software, San Diego California USA) was used for analysis.

\section{Results}

3.1. GC-MS Analysis. Separation and identification of different phytoconstituents present in the closely related genus in mature and young leaves was analyzed by GC-MS. Proportional chromatograms of unlike varieties of khat extracts are shown in Figure 1. The phytochemical composition of the young and mature extracts, including psychoactive cathine and cathinone, is shown in Table 1. The total area percentage for identified components was $63.56 \%$, $53.98 \%, 62.17 \%, 36.67 \%, 52.57 \%$, and $67.12 \%$ for N1, G1, N2, G2, N3, and G3, respectively. Cathine, a chief psychostimulant constituent, was found in all three varieties of young leaves in different proportions, viz., $0.55 \%, 0.77 \%$, and $0.67 \%$, for N1, N2, and N3, respectively, whereas, only $0.35 \%$ and $0.16 \%$ found in $\mathbf{G 1}$ and $\mathbf{G} 3$ samples, respectively. The lowest amount was found in G3 and the highest amount, $0.77 \%$, in the extract of $\mathbf{N} 2$. However, no trace of either cathine or cathinone was found in the extracts of mature G2. Cathinone was found in a lesser amount than cathine, $0.05 \%$ to $0.26 \%$, in all extracts which were studied.

3.2. Cytotoxicity Studies. The cytotoxicity of the six khat extracts was determined against three cancer cells, in addition to one normal cell line to compare the selectivity of each extract. The $\mathrm{IC}_{50}$ of $\mathrm{N} 1$ ranged from $29-56 \mu \mathrm{g} / \mathrm{mL}$ in the three cancer cell lines, and it was $30 \mu \mathrm{g} / \mathrm{mL}$ against MRC5, normal cells, which was similar to its effect on MCF7. G1 extract showed similar cytotoxicity against the three cell lines, but it was at least two-fold more toxic towards the normal cells compared with the cell lines (Table 2). N2 and G2 extracts exhibited similar activity, with the highest effect also on MRC5 normal cells. N3 extract was the most significant, as it was $4-8$ folds more cytotoxic against MRC5 cells compared with MCF7, A2780, and HT29 cells. Extract G3 was the only one to show less cytotoxicity on MRC5 cells compared to A2780 and HT29 cells.

The chemical compositions of the ethanolic extract of N1 and $\mathbf{G 1}$ are tabulated in Table 1. The ethanolic extract of $\mathbf{N} 2$ consisted of a variety of 26 chemical constituents (63.56\%) including six alkanes (11.32\%), three each of alkyl benzenes (7.88\%), monoamine alkaloids (1.28\%), and acyclic diterpenes (3.98\%), two glycosides (10.79\%), and one each of alkyl nitrile $(2.0 \%)$, aromatic ketone $(1.54 \%)$, aromatic alcohol (2.61\%), fatty ester (1.25\%), alkenyl ester (2.50\%), disaccharide (4.62\%), alkyl amide (3.59\%), and phytosterol (1.71\%). The predominant constituent detected in the $\mathbf{N} 2$ sample was 4-methyl mannitol (8.73\%) followed by 1ethenyl-3-ethyl-benzene (4.97\%), galactitol (4.62\%), 1ethenyl-4-ethyl-benzene (3.94\%), $\beta$-sitosterol (3.71\%), (Z)13-docosenamide (3.59\%), 4-methyldodecane (3.12\%), and 1-dodecanol (3.09\%). The other chemical constituents present in the $\mathbf{N} 2$ sample included 2,2' -azo bis [2-methylpropane nitrile (1.46\%), 2,2-dimethyltetradecane (2.48\%), 5methylundecane (1.54\%), 4,6-dimethyldodecane (1.23\%), 1phenyl-1,2-propane-dione (1.51\%), 1-phenylpropanol $(2.61 \%)$, cathinone $(0.22 \%)$, cathine $(0.77 \%)$, 3-methoxyamphetamine (0.29\%), 1-hydroxy-1-phenyl-2-(acetylamino) propane $(1.02 \%)$, methyl $\alpha$-D-glucopyranoside $(2.06 \%)$, heneicosane $(1.09 \%)$, isobutyl tetradec-3-enyl fumarate $(2.51 \%)$, galactitol $(4.62 \%)$, and phytol $(1.10 \%)$.

Twenty-three chemical constituents were characterized in the ethanolic extract of mature Kofat (G2) amounting $53.98 \%$. There were six alkanes present in maximum percentage (13.50\%) along with one alkyl amide (6.92\%), two alkyl benzenes (6.64\%), two glycosides $(5.08 \%)$, and two acyclic diterpenes (1.74). Various constituents, viz., alkyl nitrile (1.62\%), sesquiterpene (1.04), cycloalkane (4.28), alkyl alcohol (2.69\%), fatty ester (1.05\%), aromatic ester $(1.05 \%)$, fatty ester (3.06\%), aromatic acid (1.8\%), fluoroalkyl ester (1.46), and phytosterol (1.97\%) were detected individually. The main phytoconstituents of $\mathbf{G} 2$ were $(\mathrm{Z})$-13-docosenamide (6.92\%), 1-methyl-2-phenyl cyclopropane (4.28\%), 3methyl-5-propylnonane (3.99\%), 1-phenyl-3-ethyl-benzene (3.63\%), 4-methyl mannitol (3.27\%), 1,3-diethenyl-benzene (3.10\%), and palmitic acid (3.06\%). In addition, tetramethylbutanedinitrile (1.62\%), 2,6,10-trimethyldodecane (1.04\%), 2,6,7-trimethyldecane (1.74\%), 2,6-dimethyldecane (1.61\%), 2,7,10-trimethyldodecane (1.38\%), methyl- $\beta$-Dgalactopyranoside (1.81\%), 2,6,10,14-tetramethylhexadecane (1.55\%), dibutyl phthalate (1.05\%), phthalic acid (1.8\%), and heptacosyl heptafluorobutyrate (1.46\%) were also detected only in the $\mathbf{G} 2$ sample.

The chemical constituents characterized in both the species were 2,2,6-trimethyldecane, 1-ethenyl-3-ethyl-benzene, 1,3-diethenyl-benzene, 1-dodecanol, tetradecanyl acrylate, 4-methyl mannitol, 3,7,11,15-tetramethyl-2hexadecen-1-ol, (Z)-13-docosenamide, and $\beta$-sitosterol, and their amounts varied from 8.73 to $0.19 \%$. Three euphorigenic and psychostimulant monoamine alkaloids cathine $(0.77 \%)$, cathinone $(0.22 \%)$, and 3-methoxyamphetamine $(0.29 \%)$ were only present in young leaves.

The ethanolic extract of $\mathbf{N 2}$ consisted of 26 variety of chemical constituents (62.17\%) including six alkanes (11.86\%), three each of aromatic compounds (9.76\%) two each of sesquiterpenes (3.65\%), monoamine alkaloids $(0.81 \%)$, and one each of alkane nitrile $(1.91 \%)$, aromatic ester (1.33\%), alkyl alcohol (2.54\%), fatty acid (1.58\%), monosaccharide (6.37\%), acyclic diterpenic alcohol (1.92\%), 


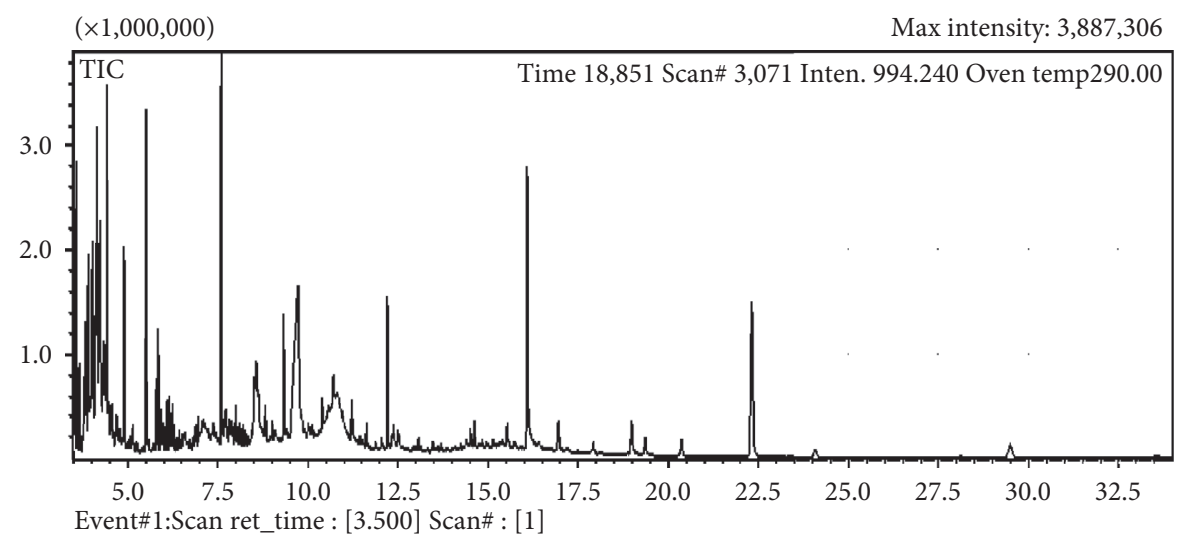

(a)

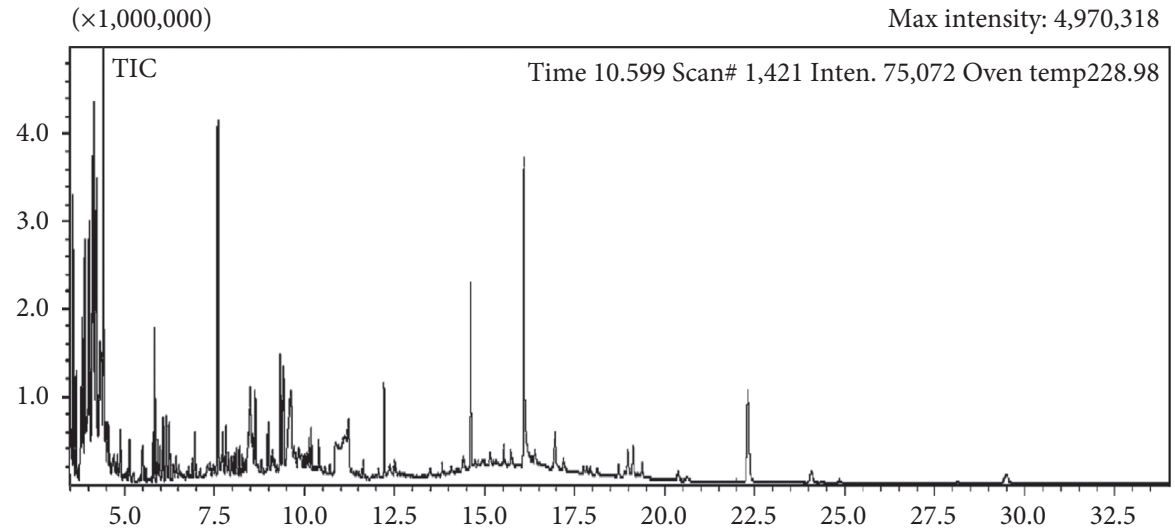

(b)

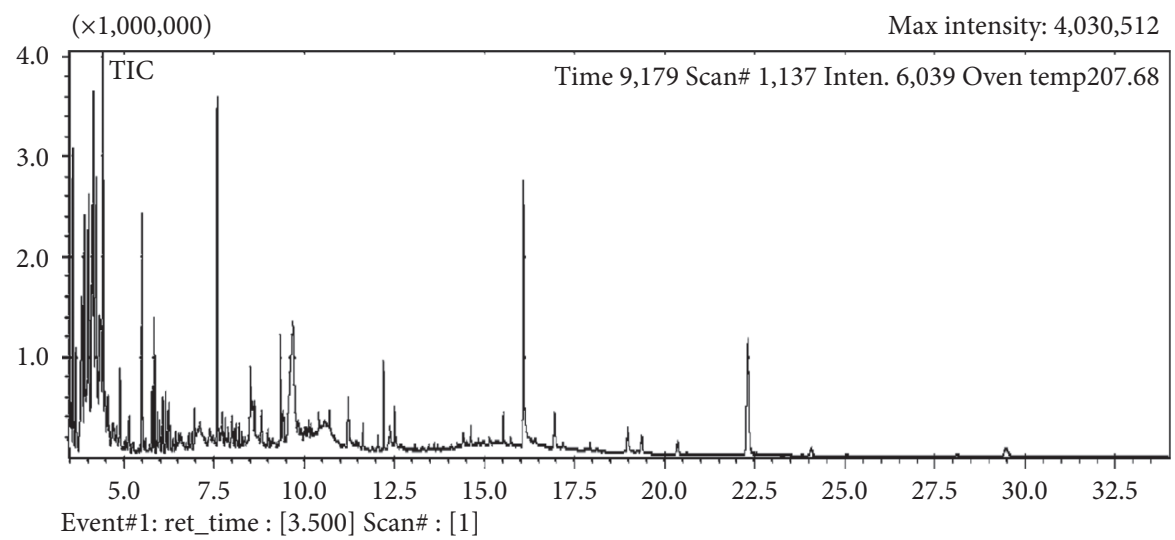

(c)

Figure 1: Representative typical GC-MS total ion current (TIC) chromatograms of three ethanolic extracts of khat samples: (a) young Kofat N1, (b) mature Kofat G1, and (c) young Gahasha N3.

diterpene (2.54\%), alkyl mercaptan (2.62\%), silyloxy alkane (1.93\%), disaccharide (3.98\%), vit. C ester (1.42\%), alkyl amide (3.95\%), and phytosterol (4.03\%). The predominant constituent detected in the $\mathbf{N} 2$ sample was 4-methyl mannitol (6.37\%) followed by $\beta$-sitosterol (4.03\%), 9-octadecenamide $(3.95 \%)$, 1-ethenyl-4-ethyl-benzene $(3.76 \%)$, 1 ethenyl-3-ethyl-benzene (3.24\%), and 3-methyl-5-propylnonane $(3 \%)$. The other chemical constituents present in the $\mathbf{N} 2$ sample included heneicosane (2.89\%), 2,2,11,11tetramethyldodecane $(2.4 \%)$, tetramethylbutane dinitrile
(1.91\%), 2,2-dimethyldecane (0.28\%), benzene $\alpha$-hydroxyethyl acetate (1.33\%), tert-nonyl mercaptan (2.62\%), and 3ethyl-6-trimethylsilyloxyoctane (1.93\%).

Twenty-seven chemical constituents were characterized in the ethanolic extract of G2 amounting 36.67\%. Monosaccharide $(7.11 \%)$ and disaccharide $(10.55 \%)$ were present in maximum amount. There were three aromatic compounds $(7.71 \%)$ and four alkanes with high percentage $(6.68 \%)$ along with two each of sesquiterpene $(2.75 \%)$ and monoamine alkaloid (0.44\%). Various constituents, viz., 
TABle 1: Chemical composition of alcoholic extract of young and mature leaves of Kofat, Gaifi, and Gahasha species.

\begin{tabular}{|c|c|c|c|c|c|c|c|c|}
\hline \multirow{2}{*}{$\begin{array}{l}\text { S. } \\
\text { no }\end{array}$} & \multirow{2}{*}{$\begin{array}{l}\text { Retention } \\
\text { time }\end{array}$} & \multirow[b]{2}{*}{ Chemical constituents } & \multicolumn{2}{|c|}{$\%$ area Kofat } & \multicolumn{2}{|c|}{$\%$ area Gaifi } & \multicolumn{2}{|c|}{$\%$ area Gahasha } \\
\hline & & & $\begin{array}{l}\text { Young } \\
\text { (N1) }\end{array}$ & $\begin{array}{c}\text { Mature } \\
(\text { G1) }\end{array}$ & $\begin{array}{l}\text { Young } \\
\text { (N2) }\end{array}$ & $\begin{array}{l}\text { Mature } \\
\text { (G2) }\end{array}$ & $\begin{array}{l}\text { Young } \\
\text { (N3) }\end{array}$ & $\begin{array}{c}\text { Mature } \\
(\text { G3) }\end{array}$ \\
\hline 1 & 3.52 & $2,2^{\prime}$-Azo bis [2-methyl-propane nitrile] & 2.0 & - & - & 1.43 & 1.88 & 2.0 \\
\hline 2 & 3.56 & Tetramethylbutanedinitrile & - & 1.62 & 1.91 & - & - & - \\
\hline 3 & 3.6 & 2,6,10-Trimethyldodecane & - & 1.04 & 0.98 & 0.66 & 1.14 & 0.95 \\
\hline 4 & 3.83 & 2,2-Dimethyldecane & - & 2.75 & 0.28 & - & 0.36 & 0.22 \\
\hline 5 & 3.83 & 2,2-Dimethyltetradecane & 2.48 & - & - & 1.77 & - & - \\
\hline 6 & 3.84 & 2,2,11,11-Tetramethyldodecane & - & - & 2.4 & - & - & - \\
\hline 7 & 3.9 & 2,2,6-Trimethyldecane & 1.86 & 2.11 & 1.83 & 1.42 & 5.34 & 4.56 \\
\hline 8 & 4.0 & 4-Methyldodecane & 3.12 & - & & & 3.86 & - \\
\hline 9 & 4.02 & 3-Methyl-5-propylnonane & - & 3.99 & 3 & 2.32 & - & 3.6 \\
\hline 10 & 4.1 & 3,6-Dimethylundecane & - & - & - & - & - & 1.41 \\
\hline 11 & 4.11 & 2,6,7-Trimethyldecane & - & 1.74 & - & - & - & - \\
\hline 12 & 4.15 & 1-Ethenyl-3-ethyl-benzene & 4.97 & 3.63 & 3.24 & 2.52 & 3.86 & - \\
\hline 13 & 4.16 & 1-Methyl-2-phenylcyclopropane & - & 4.28 & - & - & - & - \\
\hline 14 & 4.22 & 1-Methyl-1-p-tolyethyl butyrate & - & - & - & - & - & 3.77 \\
\hline 15 & 4.23 & 1-Ethenyl-4-ethyl-benzene & 3.94 & - & 3.76 & - & 4.71 & - \\
\hline 16 & 4.24 & 1-Phenyl 1-butene & - & - & - & 2.95 & - & - \\
\hline 17 & 4.33 & 5-Methylundecane & 1.54 & - & 1.46 & 1.13 & 1.93 & - \\
\hline 18 & 4.34 & 2,6-Dimethyldecane & - & 1.61 & - & - & - & - \\
\hline 19 & 4.36 & $2,7,10$-Trimethyldodecane & - & 1.38 & 2.67 & 2.09 & 2.03 & - \\
\hline 20 & 4.37 & 4,6-Dimethyldodecane & 1.23 & - & - & - & 1.5 & 1.08 \\
\hline 21 & 4.42 & 1,3-Diethenyl-benzene & 2.92 & 3.01 & 2.76 & 2.22 & 3.21 & 6.01 \\
\hline 22 & 4.9 & 1-Phenyl-1,2-propanedione & 1.51 & - & - & - & 0.72 & 0.5 \\
\hline 23 & 5.5 & 1-Phenylpropanol & 2.61 & - & - & - & - & - \\
\hline 24 & 5.5 & Benzene $\alpha$-hydroxyethyl acetate & - & - & 1.33 & - & - & - \\
\hline 25 & 5.51 & $\alpha$-Hydroxyethyl benzene acetate & - & - & - & - & 1.91 & - \\
\hline 26 & 6.6 & Cathinone & 0.22 & - & 0.26 & 0.09 & 0.16 & 0.16 \\
\hline 27 & 7.0 & Cathine & 0.77 & - & 0.55 & 0.35 & 0.67 & 0.05 \\
\hline 28 & 7.1 & 3-Methoxyamphetamine & 0.29 & - & - & - & - & - \\
\hline 29 & 7.3 & p- $\alpha$-Dimethyl phenyl ethyl amine & - & - & - & - & - & 1.9 \\
\hline 30 & 7.6 & 1-Dodecanol & 3.09 & 2.69 & 2.54 & 2.44 & 2.89 & 2.09 \\
\hline 31 & 8.4 & Methyl $\beta$-D-galactopyranoside & - & 1.81 & - & - & - & - \\
\hline 32 & 8.5 & 1-Hydroxy-1-phenyl-2-(acetylamino) propane & 1.02 & - & - & - & - & - \\
\hline 33 & 8.5 & $\begin{array}{l}\mathrm{N} \text {-Crotonyl-N-(4-methoxyphenyl)- } \\
\text { aminomalonic acid, diethyl ether }\end{array}$ & - & - & - & 1.44 & - & - \\
\hline 34 & 8.57 & Methyl $\alpha$-D-glucopyranoside & 2.06 & - & - & - & - & - \\
\hline 35 & 8.57 & Octyl- $\beta$-D-glucopyranoside & - & - & - & - & - & 1.91 \\
\hline 36 & 8.57 & Capric acid (decanoic acid) & - & - & 1.58 & 1.71 & - & - \\
\hline 37 & 8.6 & Heneicosane & 1.09 & & 2.89 & - & - & - \\
\hline 38 & 8.63 & $\begin{array}{l}\text { Methyl 6-O-[1-methylpropyl]- } \beta-\mathrm{D} \text { - } \\
\text { galactopyranoside }\end{array}$ & - & - & - & 3.74 & - & - \\
\hline 39 & 9.3 & Tetradecanyl acrylate & 1.25 & 1.05 & - & 1.31 & 1.02 & - \\
\hline 40 & 9.4 & 2,6,10,14-Tetramethylhexadecane & - & 1.55 & - & 1.13 & - & - \\
\hline 41 & 9.7 & 4-Methyl mannitol & 8.73 & 3.27 & 6.37 & 7.11 & 6.52 & 6.16 \\
\hline 42 & 10.39 & Neophytadiene & & & 2.54 & 1.26 & - & 1.18 \\
\hline 43 & 10.5 & 3,7,11,15-Tetramethyl 2-hexadecen-1-ol & 1.33 & 0.19 & 1.92 & 3.35 & - & 2.27 \\
\hline 44 & 10.7 & Isobutyl tetradec-3-enyl fumatate & 2.51 & - & - & - & - & - \\
\hline 45 & 10.8 & Galactitol & 4.62 & - & 3.98 & 10.55 & - & - \\
\hline 46 & 10.84 & Tert-nonyl mercaptan & - & - & 2.62 & - & - & - \\
\hline 47 & 10.86 & Dibutyl phthalate & - & 1.05 & - & - & - & - \\
\hline 48 & 10.92 & 3-Ethyl-6-trimethylsilyloxyoctane & - & - & 1.93 & - & - & - \\
\hline 49 & 11.12 & Palmitic acid ( $n$-hexadecanoic acid) & - & 3.06 & - & - & - & 2.95 \\
\hline 50 & 11.21 & Ascorbic acid 2,6-dihexadecanoate & - & - & 1.42 & 2.69 & - & - \\
\hline 51 & 12.2 & Phytol & 1.1 & - & - & - & - & - \\
\hline 52 & 12.52 & Stearic acid & - & - & - & - & - & 6.58 \\
\hline 53 & 14.62 & Phthalic acid (1,2-benzenedicarboxylic acid) & - & 1.8 & - & - & - & - \\
\hline 54 & 16.0 & (Z)-13-Docosenamide & 3.59 & 6.92 & - & - & 4.99 & 15.7 \\
\hline 55 & 16.08 & 9-Octadecenamide & - & - & 3.95 & 5.54 & - & - \\
\hline 56 & 16.93 & Pentatriacontane & - & - & - & - & 1.07 & - \\
\hline
\end{tabular}


TABLE 1: Continued.

\begin{tabular}{|c|c|c|c|c|c|c|c|c|}
\hline \multirow[b]{2}{*}{$\begin{array}{l}\text { S. } \\
\text { no }\end{array}$} & \multirow[b]{2}{*}{$\begin{array}{l}\text { Retention } \\
\text { time }\end{array}$} & \multirow[b]{2}{*}{ Chemical constituents } & \multicolumn{2}{|c|}{$\%$ area Kofat } & \multicolumn{2}{|c|}{$\%$ area Gaifi } & \multicolumn{2}{|c|}{$\%$ area Gahasha } \\
\hline & & & $\begin{array}{c}\text { Young } \\
\text { (N1) }\end{array}$ & $\begin{array}{c}\text { Mature } \\
(\text { G1) }\end{array}$ & $\begin{array}{c}\text { Young } \\
\text { (N2) }\end{array}$ & $\begin{array}{c}\text { Mature } \\
\text { (G2) }\end{array}$ & $\begin{array}{c}\text { Young } \\
\text { (N3) }\end{array}$ & $\begin{array}{c}\text { Mature } \\
\text { (G3) }\end{array}$ \\
\hline 57 & 16.95 & Heptacosyl heptafluorobutyrate & - & 1.46 & - & - & - & - \\
\hline 58 & 16.96 & 1-Triacontanol & - & - & - & 1.38 & - & - \\
\hline 59 & 22.3 & $\beta$-Sitosterol & 3.71 & 1.97 & 4.03 & 3.61 & 2.8 & 2.07 \\
\hline 60 & 24.04 & Lupeol & - & - & - & 1.23 & - & - \\
\hline & & tected compounds (\%) & 63.56 & 53.98 & 62.17 & 36.67 & 52.57 & 67.12 \\
\hline
\end{tabular}

TABLE 2: Cytotoxic activity of the six extracts against three cell lines and one normal fibroblast (MTT $\left.72 \mathrm{~h} \mathrm{IC}{ }_{50} \pm \mathrm{SD} \mu \mathrm{g} / \mathrm{mL}\right)$.

\begin{tabular}{|c|c|c|c|c|}
\hline \multirow{2}{*}{ Ethanolic extract of khat } & \multicolumn{4}{|c|}{$\mathrm{IC}_{50}$} \\
\hline & MCF7 & A2780 & HT29 & MRC5 \\
\hline N1 & $29.91 \pm 0.03$ & $53.78 \pm 7.45$ & $56.58 \pm 4.44$ & $30.63 \pm 1.59$ \\
\hline G1 & $29.23 \pm 0.48$ & $20.97 \pm 5.03$ & $59.33 \pm 0.74$ & $15.29 \pm 2.51$ \\
\hline N2 & $29.34 \pm 0.53$ & $52.12 \pm 4.41$ & $56.63 \pm 6.99$ & $22.94 \pm 1.20$ \\
\hline G2 & $51.11 \pm 9.05$ & $36.83 \pm 2.89$ & $59.41 \pm 0.38$ & $36.22 \pm 6.31$ \\
\hline N3 & $22.65 \pm 3.51$ & $23.74 \pm 7.35$ & $51.22 \pm 0.28$ & $6.15 \pm 0.33$ \\
\hline G3 & $54.90 \pm 4.08$ & $38.47 \pm 0.19$ & $39.22 \pm 0.10$ & $41.48 \pm 1.25$ \\
\hline
\end{tabular}

alkane nitrile $(1.43 \%)$, alkyl ester $(1.31 \%)$, acyclic norditerpene $(1.13 \%)$, alkyl alcohol $(1.38 \%)$, and pentacyclic triterpene $(1.23 \%)$ were detected individually. The main phytoconstituents of G2 were galactitol (10.55\%), 4-methyl mannitol (7.11\%), 9-octadecenamide (5.45\%), methyl 6-O[1-methylpropyl]- $\beta$-D-galactopyranoside (3.74\%), 3,7,11,15tetramethyl-2-hexadecen-1-ol (3.35\%), ascorbic acid 2,6dihexadecanoate (2.69\%), 3-methyl-5-propylnonane $(2.32 \%)$, 2,7,10-trimethyldodecane (2.09\%), 1-ethenyl-3-ethyl-benzene (2.52\%), 1-phenyl 1-butene (2.95\%), 1,3-diethenyl benzene (2.22\%), and 1-dodecanol (2.44\%). In addition, $2,2^{\prime}$-azobis [2methyl] propane nitrile $(1.43 \%), 2,2$-dimethyltetradecane (1.77\%), N-crotonyl-N-(4-methoxyphenyl)-aminomalonic acid, diethyl ether (1.44\%), tetradecanyl acrylate (1.31\%), 2,6,10,14-tetramethyl pentadecane (1.13\%), 1-triacontanol (1.38\%), and lupeol (1.23\%) were also detected in the G2 sample.

The chemical constituents characterized in both the species were 2,6,10-trimethyldodecane, 2,2,6-trimethyldecane, 3-methyl-5-propylnonane, 2,7,10-trimethyldodecane, 1-ethenyl-3-ethyl-benzene, 5-methylundecane, 1,3-diethenyl benzene, cathinone, cathine, 1-dodecanol, capric acid (decanoic acid), 3,7,11,15-tetramethyl-2-hexadecen-1-ol, 4methyl mannitol, neophytadiene, ascorbic acid 2,6-dihexadecanoate, 9-octadecenamide, and $\beta$-sitosterol, and their amounts varied from $10.55 \%$ to $0.09 \%$.

The chemical compositions of $\mathbf{N} 3$ consisted of a variety of 21 chemical constituents $(52.57 \%)$ including six alkanes $(12.56 \%)$, three each of aromatic compounds (11.78\%), two each of sesquiterpenes $(3.17 \%)$ and monoamino alkaloid $(0.83 \%)$. Alkane nitrile $(1.88 \%)$, phenyl ketone $(0.72 \%)$, aromatic ester $(1.91 \%)$, aliphatic alcohol (2.89\%), alkyl ester (1.02\%), monosaccharide (6.52\%), alkyl amide (4.99\%), and phytosterol $(2.8 \%)$ were present individually in young leaves. The predominant constituent detected in the sample was 2,2,6-trimethyldecane (5.34\%), 4-methyldodecane (3.86\%), 1-ethenyl-3-ethyl benzene (3.86\%), 1-ethenyl-4-ethyl benzene (4.71\%), 1,3-diethenyl benzene (3.21\%), 4-methyl mannitol (6.52\%), and (Z)-13-docosenamide (4.99\%). The other chemical constituents present in the N3 sample included 2,2'-azobis [2-methyl]-propane nitrile (1.88\%), 2,6,10-trimethyldodecane $(1.44 \%), \quad 2,2$-dimethyldecane (0.36\%), 5-methylundecane (1.93\%), 2,7,10-trimethyldodecane $(2.03 \%), 4,6$-dimethyldodecane $(1.5 \%)$, 1-phenyl 1,2propanedione $(0.72 \%)$, cathinone $(0.16 \%)$, cathine $(0.67 \%)$, 1 -dodecanol $(2.89 \%)$, tetradecanyl acrylate $(1.02 \%)$, pentatriacontane $(1.07 \%)$, and $\beta$-sitosterol $(2.8 \%)$.

Twenty-two chemical constituents were characterized in the ethanolic extract of mature Gahasha (G3) amounting $67.12 \%$. There were five alkanes present in maximum percentage $(10.87 \%)$ along with two alkyl amides $(17.6 \%)$, monoamino alkaloid (0.22\%), and fatty acid (9.53\%). Alkane nitrile $(2 \%)$, sesquiterpene $(0.95 \%)$, aromatic ester $(3.77 \%)$, aromatic compound (6.01\%), phenyl ketone (0.5\%), alkyl amide (1.9\%), aliphatic alcohol (2.89\%), glucoside (1.91\%), monosaccharide (6.16\%), diterpene (1.18\%), diterpenol (2.27\%), and phytosterol (2.07\%) were detected individually. The main phytoconstituents of leaves were (Z)-13-docosenamide (15.7\%), 2,2,6-trimethyldecane (4.56\%), 3-methyl-5propylnonane (3.6\%), 1-methyl-1-p-tolyethyl butyrate (3.77\%), 1,3-diethenyl benzene (6.01\%), 4-methyl mannitol $(6.16 \%)$, and stearic acid (6.58\%). In addition, $2,2^{\prime}$-azobis [2methyl]-propane nitrile (2\%), 2,6,10-trimethyldodecane (0.95\%), 2,2-dimethyldecane $(0.22 \%), 3,6$-dimethylundecane $(1.41 \%), 4,6$-dimethyldodecane $(1.08 \%)$, 1-phenyl 1,2propanedione $(0.5 \%)$, cathinone $(0.17 \%)$, cathine $(0,05 \%)$, p- $\alpha$-dimethyl phenyl ethyl amine (1.9\%), 1-dodecanol (2.89\%), octyl- $\beta$-D-glucopyranoside (1.91\%), neophytadiene (1.18\%), 3,7,11,15-tetramethyl 2-hexadecen-1-ol (2.27\%), palmitic acid $(2.95 \%)$, and $\beta$-sitosterol $(2.07 \%)$ were also detected in the sample. The chemical constituents characterized in both the species were 2,6,10-trimethyldodecane, 
2,2-dimethyldecane, 2,2,6-trimethyldecane, 2,2' -azobis [2methyl]-propane nitrile, 4,6-dimethyldodecane, 1,3-diethenyl benzene, 1-phenyl 1,2-propanedione, 1-dodecanol, 4methyl mannitol, (Z)-13-docosenamide, and $\beta$-sitosterol, and their amounts varied from $15.7 \%$ to $0.05 \%$. Two euphorigenic and psychostimulant monoamine alkaloid cathine $(0.67 \%)$ were found in young leaves and in minor traces in mature leaves; cathinone was present in both types of leaves in almost the same concentrations.

Chemometric multivariate analysis using hierarchical cluster analysis (HCA) was performed using the nearest and furthest neighbor cluster methods to analyze and classify the six samples of extracts based on the 60 different components detected in these samples.

Based on GC-MS results and the hierarchical cluster analysis in the table of the agglomeration schedule (Table 3) for cluster solution, there is a sudden jump/gap in the distance coefficient. The solution before the gap indicated a good solution. Accordingly, we could determine two major clusters of samples based on the furthest neighbor clustering method. The first cluster consists of N1, N3, N2 , and G2, and the second cluster consists of G1 and G3. In the first cluster, N1 and N3 combined and showed similar characteristics and therefore can be included in the same subcluster leaving $\mathbf{N 2}$ and $\mathbf{G 2}$ forming the second subcluster in this first group. The hierarchical cluster analysis results are shown in Table 3 and Figure 2.

In previous cytotoxicity studies [20,21], the methanolic extract of Catha edulis showed cytotoxicity on MCF 7 and HL60 cells with $\mathrm{IC}_{50} 33-200 \mu \mathrm{g} / \mathrm{ml}$, respectively. However, the effect of those extracts was not tested in normal cells to evaluate the possible adverse effect of chewed khat on the health of normal living cells. Thus, khat extracts in this study showed comparable cytotoxicity on the cancer cells, but more interestingly, they were more cytotoxic against the normal cells, which could be associated with the different mouth cavity lesions.

\section{Discussion}

Out of total 26, 26, and 20 chemical constituents present in ethanolic extracts of young leaves, 6, 4, and 2 different chemical entities were found in $\mathbf{N 1}, \mathbf{N} 2$, and N3, respectively. These chemical compounds were not found in other extracts and were unique for them. $\mathbf{N} 1$ contains six unique compounds: 1-phenyl propanol, cathine, 1-hydroxy-1-phenyl-2-(acetylamino) propane, methyl $\alpha$-Dglucopyranoside, isobutyl tetradec-3-enyl fumarate, and phytol. N1 has almost equipotent cytotoxic activity on both normal MRCS (30.63) and human breast adenocarcinoma, MCF7 (29.91), and observed the least activity on HT29. It has no advantage over cancerous cells. N2 has four unique compounds: 2,11,11-tetramethyldodecane, benzene $\alpha$-hydroxyethyl acetate, tert-nonyl mercaptan, and 3-ethyl-6-trimethylsilyloxyoctane. It is more cytotoxic to normal cells than MCF7. N3 has $\alpha$-hydroxy ethyl benzene acetate and pentatriacontane. It was cytotoxic compared to all other extracts, and was found that its activity is more on normal cells. $\mathbf{N} \mathbf{1}$ was the least cytotoxic among the three, and N3 is the most toxic among all extracts.

Similarly, we found some rare compounds in the extracts of mature leaves. G1, G2, and G3 have 7, 5, and 4 of such uncommon constituents in them, respectively. G1 contains seven such unique compounds: 2,6,7-trimethyldecane, 1methyl-2-phenylcyclopropane, 2,6-dimethyldecane, methyl $\beta$-D-galactopyranoside, dibutyl phthalate, phthalic acid (1,2benzenedicarboxylic acid), and heptacosyl heptafluorobutyrate. It is more cytotoxic to normal cells than other cancerous cells. G2 has five unique compounds: 1phenyl 1-butene, N-crotonyl-N-(4-methoxyphenyl)-aminomalonic acid diethyl ether, methyl 6-O-[1-methylpropyl]$\beta$-D-galactopyranoside, 1-triacontanol, and lupeol. It has almost similar cytotoxic activity on both normal and A2780 cells but observed the least effect on HT29 cells. G3 contains four rare compounds: 3,6-dimethylundecane, 1-methyl-1-ptolyethyl butyrate, $\mathrm{p}$ - $\alpha$-dimethyl phenylethylamine, and stearic acid. It also has almost similar cytotoxic activity in both normal and cancerous cells, but the minimum activity was observed in HT29 cells.

No compounds were common between $\mathbf{N} \mathbf{1}$ and $\mathbf{G} \mathbf{1}$ or N3 and G3 but two common chemical constituents in N2 and G2 extracts were observed: 1-hydroxy-1-phenyl-2(acetylamino) propane and 9-octadecenamide. Similarly, sixteen unique compounds were present in $\mathrm{N1}$, and none were found in $\mathbf{G 1}$; thirteen individual compounds were found in G1, but none observed in N1. N2 has six unique compounds, but the same was not found in G2; G2 has nine other distinct compounds that were absent in $\mathbf{N 2}$. N3 has eight unique compounds, but they were not found in G3; however, G3 has ten other compounds that were absent in N3. The extracts of mature and young leaves have an almost different set of chemical constituents. It could be the reason why there is a difference in their cytotoxicity. Further study is required to identify the exact role of each of these chemical constituents found in these extracts.

In terms of the presence of cathinone and cathine, we found that the maximum content of cathinone in $\mathbf{N} 1(0.22)$ and N2 (0.26) and N3 is only 0.16. Cathinone was not found in mature leaves G1 but was found in G2 and G3 (0.09 and 0.16 , respectively). Similarly, the presence of cathine was less in mature leaves G2 (0.35) and G3 (0.05) but was not found in mature G1 compared to young leaves N1. The maximum cathine was found in N1 (0.77). Methoxyamphetamine (0.29) was found only in the $\mathrm{N} 1$ extract. It could be the reason for the variation in psychostimulant activities in young and mature leaves.

Isolating and characterizing the different chemical constituents in the ethanolic extract of both mature and young leaves of Catha edulis and matching its cytotoxic activity is the strength of this study; however, identifying the biological activities of each constituent found in these extracts requires further study. 
TABLe 3: Agglomeration schedule.

\begin{tabular}{|c|c|c|c|c|c|c|}
\hline \multirow{2}{*}{ Stage } & \multicolumn{2}{|c|}{ Cluster combined } & \multirow{2}{*}{ Coefficients } & \multicolumn{2}{|c|}{ Stage cluster first appears } & \multirow{2}{*}{ Next stage } \\
\hline & Cluster 1 & Cluster 2 & & Cluster 1 & Cluster 2 & \\
\hline 1 & 1 & 5 & 77.860 & 0 & 0 & 4 \\
\hline 2 & 3 & 4 & 91.668 & 0 & 0 & 4 \\
\hline 3 & 2 & 6 & 124.046 & 0 & 0 & 5 \\
\hline 4 & 1 & 3 & 126.395 & 1 & 2 & 5 \\
\hline 5 & 1 & 2 & 167.067 & 4 & 3 & 0 \\
\hline
\end{tabular}

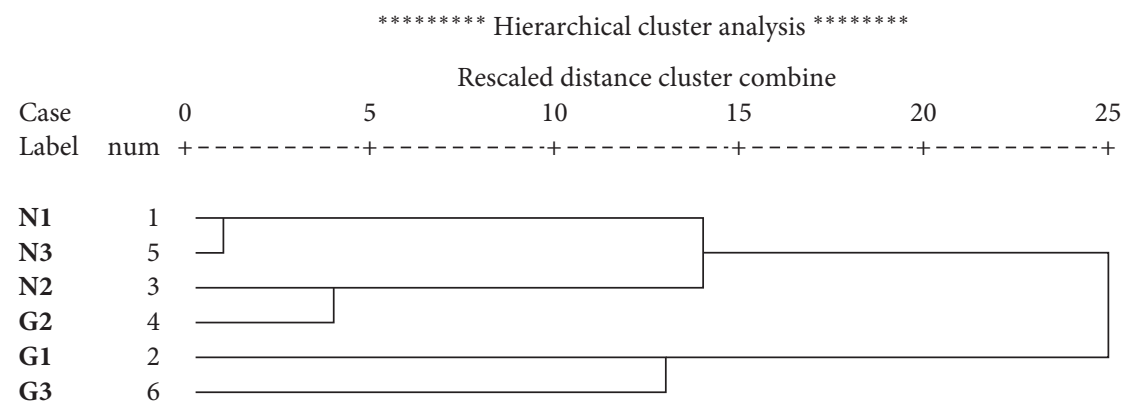

Figure 2: Dendrograms using complete linkage illustrating the average linkage between groups of complexes (hierarchical cluster analysis and rescaled distance cluster combine) for compounds detected by GC-MS in the six khat leaves extracts under study.

\section{Conclusions}

GC-MS investigations revealed and identified several remarkable phytochemicals with significant variations among them in the young and mature ethanolic extracts of the three khat cultivars. The study confirmed the presence of psychoactive cathine and cathinone in high quantities in the young leaves compared to matured leaves. Ethanolic extracts of khat showed significant cytotoxicity, $\mathrm{IC}_{50}$ ranging from $22-59 \mu \mathrm{g} / \mathrm{mL}$ on the cancer cells, compared to previous claims ( $\mathrm{IC}_{50 \text { : }}$ ranging from $33-200 \mu \mathrm{g} / \mathrm{mL}$ ); however, these extracts were also exhibiting cytotoxicity against the normal cells (MRC5 $\mathrm{IC}_{50}: 6-41 \mu \mathrm{g} / \mathrm{mL}$ ). Hence, the substantial cytotoxic effect on normal cells may pose many hazards to the health of khat consumers. Therefore, awareness campaigns on stopping the usage of khat should be implemented and facilitated in affected societies.

\section{Data Availability}

The data used to support the findings of this study are available from the corresponding author upon request.

\section{Conflicts of Interest}

All the authors declare that there are no conflicts of interest.

\section{Acknowledgments}

Authors are thankful to the Deanship of Scientific Research, Jazan University, for providing financial support (Research Group: RG-2-8) to conduct this research work. The authors are also thankful to Mr. Hamad Hakami for his technical assistance in this study.

\section{References}

[1] T. Ketema, K. Bacha, E. Alemayehu, and A. Ambelu, "Incidence of severe malaria syndromes and status of immune responses among khat chewer malaria patients in Ethiopia," PLoS One, vol. 10, no. 7, Article ID e0131212, 2015.

[2] G. Ayano, K. Yohannis, and M. Abraha, "Epidemiology of khat (Catha edulis) consumption among university students: a meta-analysis," BMC Public Health, vol. 19, no. 1, p. 150, 2019.

[3] L. Ongeri, F. Kirui, E. Muniu et al., "Khat use and psychotic symptoms in a rural khat growing population in Kenya: a household survey," BMC Psychiatry, vol. 19, no. 1, p. 137, 2019.

[4] A. Alsalahi, M. A. Alshawsh, R. Mohamed et al., "Conflicting reports on the role of the glycemic effect of Catha edulis (khat): a systematic review and meta-analysis," Journal of Ethnopharmacology, vol. 186, pp. 30-43, 2016.

[5] B. Geresu, "Khat (Catha edulis F.) and cannabinoids: parallel and contrasting behavioral effects in preclinical and clinical studies," Pharmacology Biochemistry and Behavior, vol. 138, pp. 164-173, 2015.

[6] W. Bedada, F. De Andrés, E. Engidawork et al., "The psychostimulant khat (Catha edulis) inhibits CYP2D6 enzyme activity in humans," Journal of Clinical Psychopharmacology, vol. 35, no. 6, pp. 694-699, 2015.

[7] S. T. Kimani, N. B. Patel, and P. G. Kioy, "Memory deficits associated with khat (Catha edulis) use in rodents," Metabolic Brain Disease, vol. 31, no. 1, pp. 45-52, 2016.

[8] S. I. Abdelwahab, R. M. Alsanosy, B. E. A. Rahim et al., "Khat (Catha edulis forsk.) dependence potential and pattern of use in Saudi Arabia," Biomed Research International, vol. 2015, Article ID 604526, 8 pages, 2015.

[9] S. El-Zaemey, J. Schüz, and M. Leon, "Qat chewing and risk of potentially malignant and malignant oral disorders: a systematic review," The International Journal of Occupational and Environmental Medicine, vol. 6, no. 3, pp. 129-143, 2015. 
[10] H. Yildiz, M. Komuta, C. Monsalve, P. Starkel, and C. Lefebvre, "To chew or not to chew: that's the question," Acta Clinica Belgica, vol. 71, no. 3, pp. 187-189, 2016.

[11] A. Nyongesa, J. Oduma, M. Al'Absi, and S. Chirwa, "Immunohistochemical localization of anterior pituitary cell types of vervet monkey (Chlorocebus aethiops) following subchronic cathinone exposure," Journal of Ethnopharmacology, vol. 174, pp. 168-177, 2015.

[12] M. S. Mahfouz, B. E. A. Rahim, Y. M. H. Salon et al., "Khat chewing habits in the population of the Jazan region, Saudi Arabia: prevalence and associated factors," PLoS One, vol. 10, no. 8, Article ID e0134545, 2015.

[13] M. F. A. Quadri, F. Alharbi, A. M. S. Bajonaid, I. H. Y. Moafa, A. A. Sharwani, and A. H. A. Alamir, "Oral squamous cell carcinoma and associated risk factors in Jazan, Saudi Arabia: a hospital based case control study," Asian Pacific Journal of Cancer Prevention, vol. 16, no. 10, pp. 4335-4338, 2015.

[14] A. El-Menyar, A. Mekkodathil, H. Al-Thani, and A. AlMotarreb, "Khat use: history and heart failure," Oman Medical Journal, vol. 30, no. 2, pp. 77-82, 2015.

[15] A. M. Lemieux, B. Li, and M. Al'Absi, "Khat use and appetite: an overview and comparison of amphetamine, khat and cathinone," Journal of Ethnopharmacology, vol. 160, pp. 7885, 2015.

[16] T. M. Al-Qirim, M. Shahwan, K. R. Zaidi, Q. Uddin, and N. Banu, "Effect of khat, its constituents and restraint stress on free radical metabolism of rats," Journal of Ethnopharmacology, vol. 83, no. 3, pp. 245-250, 2002.

[17] M. M. Bkhaitan, A. Z. Mirza, A. N. Abdalla et al., "Reprofiling of full-length phosphonated carbocyclic $2^{\prime}$-oxa- $3^{\prime}$-aza-nucleosides toward antiproliferative agents: synthesis, antiproliferative activity, and molecular docking study," Chemical Biology \& Drug Design, vol. 90, no. 5, pp. 679-689, 2017.

[18] S. Tan, Y. Sagara, Y. Liu, P. Maher, and D. Schubert, "The regulation of reactive oxygen species production during programmed cell death," The Journal of Cell Biology, vol. 141, no. 6, pp. 1423-1432, 1998.

[19] H. E. Soufi, M. Kameswaran, and T. Malatani, "Khat and oral cancer," The Journal of Laryngology \& Otology, vol. 105, no. 8, pp. 643-645, 1991.

[20] N. Worku, A. Mossie, A. Stich et al., "Evaluation of the in vitro efficacy of artemisia annua, rumex abyssinicus, and catha edulis forsk extracts in cancer and trypanosoma brucei cells," ISRN Biochemistry, vol. 2013, Article ID 910308, 10 pages, 2013.

[21] E. A. O. Dimba, B. T. Gjertsen, T. Bredholt et al., "Khat (Catha edulis)-induced apoptosis is inhibited by antagonists of caspase-1 and-8 in human leukaemia cells," British Journal of Cancer, vol. 91, no. 9, pp. 1726-1734, 2004. 